Baltic Astronomy, vol. 5, 319-335, 1996.

\title{
INTERSTELLAR EXTINCTION IN THE DARK CLOUD KHAVTASSI 217 IN CASSIOPEIA
}

\author{
A. Kazlauskas \\ Institute of Theoretical Physics and Astronomy, Goštauto 12, \\ Vilnius 2600, Lithuania
}

Received December 31, 1995.

\begin{abstract}
The Vilnius photometric system has been applied for the investigation of interstellar extinction in the area of dark cloud Khavtassi 217 in Cassiopeia. On the ground of photoelectric photometry of 152 stars, their photometric spectral types, absolute magnitudes, color excesses, interstellar extinctions and distances were determined. The area is divided into two regions with different extinction versus distance run. Distances of the dark clouds are determined.
\end{abstract}

Key words: interstellar medium: extinction - methods: observational - techniques: photometric

\section{INTRODUCTION}

The investigated area with the coordinates $\alpha(2000)=0^{\mathrm{h}} 50^{\mathrm{m}}$ $1^{\mathrm{h}} 30^{\mathrm{m}}, \delta(2000)=65^{\circ} 40^{\prime}-67^{\circ} 40^{\prime}$, close to the bright star $\psi$ Cas, is situated slightly above the galactic plane (galactic coordinates of the center: $\left.l=124.5^{\circ}, b=3.9^{\circ}\right)$. On photographs of the area, a complex of dark clouds is seen. In the Khavtassi (1960) atlas of the galactic dark clouds, the area coincides with the dark cloud 217. In the Lynds (1962) catalog of dark clouds the identification is very uncertain: the L1305 and L 1307 have similar galactic coordinates. This region is almost uninvestigated photometrically. Only a few stars of specific spectral types have been measured in the $U B V$ (Neckel 1974, Haupt \& Schroll 1974, Pesch 1963, Oja 1991) and uvby (Perry \& Johnston 1982, Olsen 1983) photometric systems. The spectral types and interstellar extinction are also known only for some stars. 


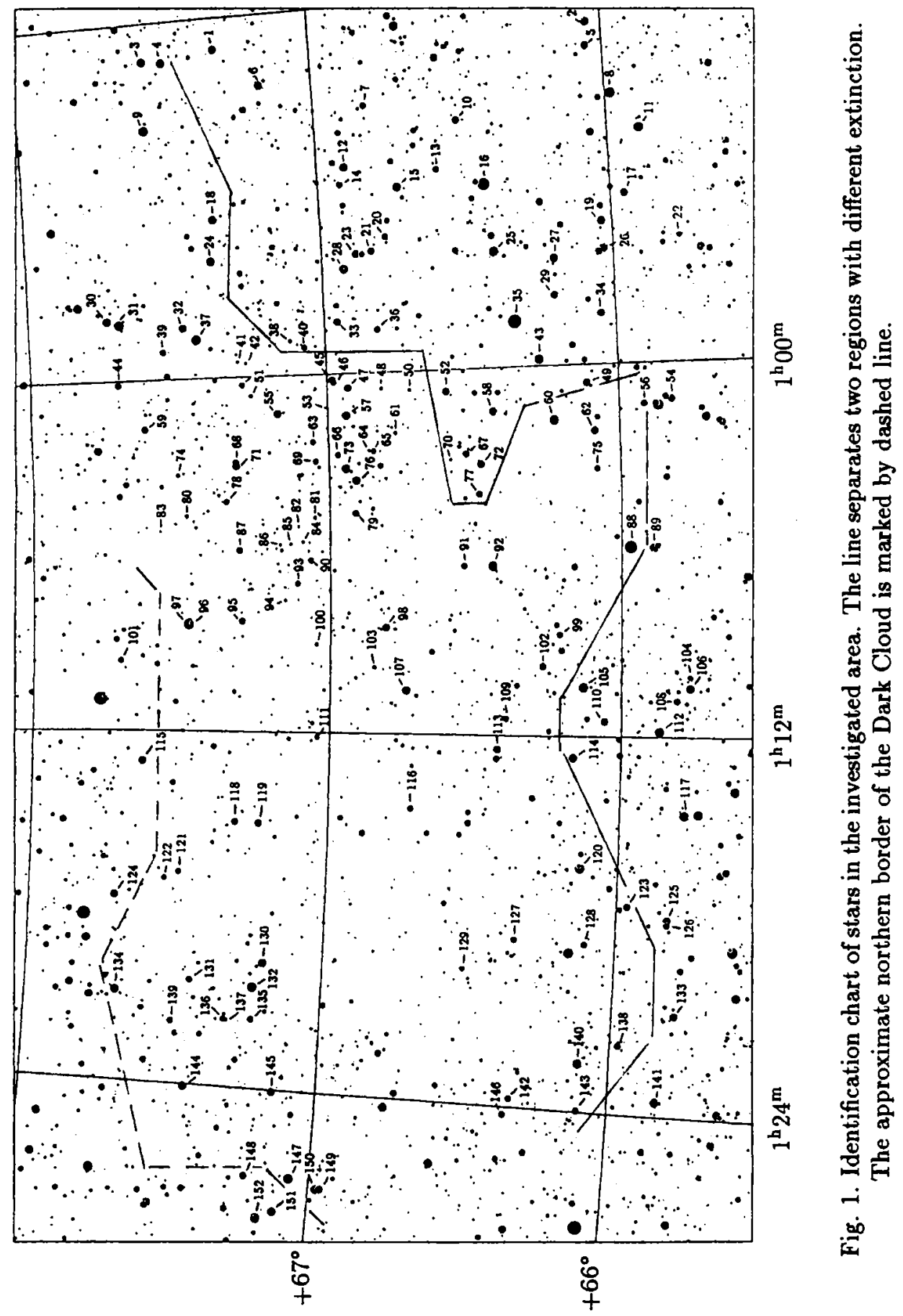


A new catalogue of extinction data (Guarinos 1995) includes just one star from the investigated area. To fill this gap, the photometric investigation of this area has been started. All the brightest stars with BD numbers in a selected field of $2^{\circ} \times 4^{\circ}$ size were measured. Trying to penetrate deeper into the dark cloud and to determine the dependence of interstellar extinction on distance, about 30 fainter stars down to $V=12.5$ mag were measured additionally in a smaller area of about 1 square degree size.

\section{PHOTOMETRIC OBSERVATIONS AND CLASSIFICATION OF STARS}

The observations were obtained in September 1992 with the 1 meter telescope of the Institute of Theoretical Physics and Astronomy at the Maidanak Observatory in Uzbekistan. A standard set of filters of the Vilnius photometric system has been used. The observed magnitudes and color indices were transformed to outside the atmosphere by Nikonov's method modified by Zdanavičius (1975). The extinction star HD 189796 (G0 V, $V=7.82$ ) has been used. Transformation equations from the instrumental to the standard system have been determined from observations of 29 stars of various spectral types and luminosities from the Cygnus Standard Region (Zdanavičius \& Černienè 1985).

The results of photometry of 152 stars are presented in Table 1 which contains: star numbers identified in Fig. 1, BD numbers, coordinates, $V$ magnitudes and color indices $U-V, P-V, X-V, Y-V$, $Z-V$ and $V-S$ in the Vilnius system, the number of independent observations. $90 \%$ of the stars were measured twice. The typical mean square errors of the magnitudes and color indices are of the order of \pm 0.01 to $\pm 0.02 \mathrm{mag}$ (for stars fainter than $V=11 \mathrm{mag}$ ). Some red stars were observed with lower accuracy in the ultraviolet passbands due to insufficient photon statistics. If the errors are greater than $\pm 0.05 \mathrm{mag}$, the indices are not given. If the errors are between \pm 0.03 and $\pm 0.05 \mathrm{mag}$, the indices are marked by colons.

Photometric spectral classes, luminosity classes and absolute magnitudes of the stars have been determined from the photometric data by using the computer program CLASS written by Vansevičius $\&$ Bridžius (1994). The quantification of stars is based on a threedimensional (spectral class, absolute magnitude and color excess) fitting of the observed color indices of the program star with those from the bank of standard stars. This program gives also the stan- 
dard deviation $\sigma$ of color indices between the program star and the closest standard star; this can be used as a measure of the classification accuracy. On the other hand, $\sigma$ values depend on spectral classes and luminosity classes: if $\sigma= \pm 0.03$ is a good accuracy for Ktype stars, it is too bad for B-type stars. To control the accuracy of the presented classification, three more methods have been applied: (a) the method of comparison of ten reddening-free $Q$-parameters of the program star with those of about 7000 stars from the General Photometric Catalogue of Stars Observed in the Vilnius System (Straižys \& Kazlauskas 1993); (b) Q, Q-diagrams calibrated in terms of spectral class and absolute magnitude by Straižys et al. (1982); (c) the probability method proposed by Zdanavicius et al. (1994).

All four methods give very similar results if the accuracy of color indices is of the order of 0.01 mag. Comparing the results of the classification obtained by different methods and taking into account the values of $\sigma$ from the CLASS program, the results of two-dimensional classification were divided into three groups according to their weight. $W=3$ means that the classification is good, i.e. all methods give very similar results and $\sigma$ is of the order of $\pm(0.01-0.02)$ mag. For $W=2$ the classification is reliable, i.e. spectral classes obtained by different methods may differ by $2-3$ spectral subclasses and $\sigma$ is of the order of $\pm(0.02-0.03)$ mag. For $W=1$ the classification is uncertain. When $\sigma$ is greater than $\pm 0.03 \mathrm{mag}$, spectral classes and absolute magnitudes may contain significant errors.

For stars Nos. 1, 91 and 93 the classification failed. Star No. 1 probably is a peculiar or an unresolved binary. Stars Nos. 91 and 93 were measured with insufficient accuracy.

The color excesses $E_{Y-V}$ were calculated as differences between the observed color indices $Y-V$ and the intrinsic color indices $(Y-V)_{0}$ of the corresponding spectral class and luminosity (Straižys 1992). 
Table 1. Results of photometry.

\begin{tabular}{|c|c|c|c|c|c|c|c|c|c|c|}
\hline $\mathrm{BD}$ & $\begin{array}{l}\alpha(2000) \\
\mathbf{h} \mathbf{m} \text { s }\end{array}$ & $\begin{array}{c}\delta(2000) \\
0\end{array}$ & $V$ & $U-V$ & $P-V$ & $X-V$ & $Y-V$ & $Z-V$ & $V-S$ & $n$ \\
\hline 1. $+66^{\circ} 61$ & 04902 & +6719.5 & 10.31 & 4.05: & 3.37 & 2.43 & 1.01 & 0.44 & 0.97 & 2 \\
\hline 2. $+66^{\circ} 97$ & 04910 & +6603.1 & 9.74 & 2.44 & 1.79 & 1.05 & 0.43 & 0.18 & 0.36 & 2 \\
\hline 3. $+66^{\circ} 62$ & 04914 & +6734.2 & 9.72 & 6.20: & 5.31: & 3.74 & 1.47 & 0.61 & 1.29 & 2 \\
\hline 4. $+66^{\circ} 63$ & 04920 & +6730.3 & 9.19 & 5.46 & 4.62 & 3.19 & 1.23 & 0.52 & 1.11 & 2 \\
\hline 5. $+65^{\circ} 100$ & 04955 & +6603.3 & 10.01 & 2.27 & 1.74 & 1.17 & 0.49 & 0.18 & 0.48 & 2 \\
\hline $6 .+66^{\circ} 64$ & 05018 & +6710.6 & 9.55 & 2.65 & 1.77 & 1.00 & 0.50 & 0.18 & 0.41 & 2 \\
\hline 7. $+66^{\circ} 65$ & 05115 & +6649.8 & 10.08 & 2.27 & 1.72 & 1.11 & 0.48 & 0.18 & 0.43 & 2 \\
\hline 8. $+65^{\circ} 101$ & 05126 & +6558.8 & 8.03 & 4.18 & 3.55 & 2.42 & 0.89 & 0.39 & 0.84 & 2 \\
\hline $9 .+66^{\circ} 66$ & 05133 & +6734.8 & 8.61 & 6.45 & 5.51 & 3.85 & 1.49 & 0.66 & 1.37 & 2 \\
\hline 10. $+65^{\circ} 102$ & 05158 & +6631.2 & 9.63 & 2.24 & 1.60 & 0.87 & 0.36 & 0.14 & 0.32 & 2 \\
\hline 11. $+65^{\circ} 103$ & 05236 & +6553.5 & 8.32 & 2.00 & 1.41 & 0.61 & 0.24 & 0.09 & 0.16 & 2 \\
\hline 12. $+66^{\circ} 67$ & 05 & +66 & 9.94 & 1.99 & 1.56 & 1.09 & 0.57 & 0.19 & 0.46 & 2 \\
\hline 13. $+65^{\circ} 104$ & 05330 & +6635.9 & 10.52 & 4.24: & 3.66 & 2.54 & 1.01 & 0.39 & 0.91 & 2 \\
\hline 14. $+66^{\circ} 68$ & 05349 & +6655.6 & 10.12 & 2.43 & 1.96 & 1.39 & 0.56 & 0.21 & 0.56 & 2 \\
\hline 15. $+65^{\circ} 105$ & 05401 & +6644.1 & 8.97 & 2.95 & 2.10 & 1.31 & 0.59 & 0.22 & 0.53 & 2 \\
\hline 16. $+65^{\circ} 106$ & 05 & +6626.1 & 7.04 & 2.19 & 1.61 & 1.00 & 0.42 & 0.17 & 0.39 & 2 \\
\hline 17. $+65^{\circ} 107$ & 05438 & +6557.2 & 10.04 & 2.55 & 1.99 & 1.34 & 0.57 & 0.23 & 0.55 & 2 \\
\hline 18. $+66^{\circ} 69$ & 05442 & +6721.9 & 9.02 & 2.36 & 1.86 & 1.29 & 0.53 & 0.21 & 0.51 & 2 \\
\hline 19. $+65^{\circ} 108$ & 05528 & +6602.3 & 9.38 & 2.28 & 1.71 & 1.11 & 0.47 & 0.17 & 0.45 & 2 \\
\hline 20 & 05533 & +6647.1 & 10.38 & 2.56 & 1.98 & 1.36 & 0.60 & 0.22 & 0.54 & 2 \\
\hline 21. $+66^{\circ} 70$ & 05600 & +66 & 10.04 & 2.74 & 2.00 & 1.25 & 0.57 & 0.20 & 0.52 & 2 \\
\hline $22 .+65^{\circ} 109$ & 05602 & +6546.8 & 11.37 & & & 2.89 : & 1.10: & 0.46 & 1.01 & 2 \\
\hline $23 .+66^{\circ} 72$ & 05604 & +6653.1 & 9.73 & 2.47 & 1.91 & 1.31 & 0.55 & 0.23 & 0.52 & 2 \\
\hline 24. $+66^{\circ} 71$ & 05604 & +6722.6 & 9.35 & 2.70 & 1.92 & 1.21 & 0.63 & 0.21 & 0.49 & 2 \\
\hline 25. $+65^{\circ} 110$ & 05615 & +6624.8 & 8.31 & 1.35 & 1.01 & 0.56 & 0.29 & 0.10 & 0.20 & 2 \\
\hline $26 .+65^{\circ} 111$ & 05618 & +6601.9 & 9.28 & 3.50 & 2.73 & 1.88 & 0.88 & 0.32 & 0.81 & 1 \\
\hline $27 .+65^{\circ} 112$ & 05632 & +6612.3 & 9.09 & 2.56 & 1.70 & 0.86 & 0.42 & 0.14 & 0.33 & 2 \\
\hline $28 .+66^{\circ} 73$ & 05632 & +6655.6 & 9.45 & 4.36 & 3.67 & 2.52 & 0.99 & 0.39 & 0.89 & 2 \\
\hline $29 .+65^{\circ} 113$ & 05741 & +6612.5 & 10.27 & 4.09: & 3.37: & 2.41 & 1.05 & 0.41 & 0.97 & 2 \\
\hline $30 .+66^{\circ} 74$ & 05756 & +6744.4 & 9.10 & 2.22 & 1.59 & 0.91 & 0.39 & 0.15 & 0.35 & 2 \\
\hline 31. $+66^{\circ} 75$ & & +6 & 8.51 & 6.66 & 5.52 & 3.93 & 1.57 & 0.66 & 1.46 & 2 \\
\hline $32 .+66^{\circ} 76$ & 05815 & +6729.0 & 9.89 & 2.24 & 1.73 & 1.17 & 0.50 & 0.19 & 0.47 & 2 \\
\hline $33 .+66^{\circ} 77$ & 05816 & +6657.4 & 9.67 & 2.35 & 1.68 & 0.91 & 0.39 & 0.13 & 0.32 & 2 \\
\hline $34 .+65^{\circ} 114$ & 05821 & +6603.2 & 9.65 & 2.46 & 1.74 & 0.87 & 0.39 & 0.14 & 0.28 & 2 \\
\hline $35 .+65^{\circ} 115$ & 05831 & +6621.1 & 5.95 & 1.66 & 1.16 & 0.45 & 0.18 & 0.07 & 0.12 & 2 \\
\hline 36. $+66^{\circ} 78$ & 05835 & +6649.4 & 10.10 & 2.34 & 1.67 & 0.94 & 0.40 & 0.15 & 0.34 & 2 \\
\hline 37. $+66^{\circ} 79$ & 05840 & +6726.4 & 8.34 & 2.25 & 1.66 & 1.02 & 0.44 & 0.17 & 0.40 & 2 \\
\hline 38 . & 05851 & +6707.3 & 11.45 & 3.09 & 2.27 & 1.43 & 0.67 & 0.25 & 0.55 & 2 \\
\hline $39 .+66^{\circ} 80$ & 05903 & +6733.4 & 10.46 & 4.00 & 3.33 & 2.35 & 0.99 & 0.40 & 0.93 & 2 \\
\hline 40 . & 05906 & +6704.2 & 10.94 & 2.39 & 1.91 & 1.32 & 0.56 & 0.23 & 0.54 & 2 \\
\hline
\end{tabular}


Table 1 (continued)

\begin{tabular}{|c|c|c|c|c|c|c|c|c|c|c|c|}
\hline No. & $\mathrm{BD}$ & $\begin{array}{c}(2000) \\
m \quad s\end{array}$ & $\delta(2000)$ & $V$ & $I-V$ & & $Y-V$ & & $Z-V$ & $V-S$ & \\
\hline 41. & & 5928 & +6718.2 & & & & & & & & 2 \\
\hline & & & & & & & & & & & \\
\hline & & & +6616.3 & 8.93 & & & & & & & \\
\hline & & & +6742.6 & 10.69 & & & & & & & \\
\hline 45. & & & & & & & & & & & 2 \\
\hline 46. & & & & & & & & & & & $?$ \\
\hline 47. & & & & 9.93 & & & & & & & 2 \\
\hline 48. & & & & & & & & & & & 2 \\
\hline 49. & & & & & & & & & & & 2 \\
\hline 50. & & & +66 & 11.95 & & & & & & & 2 \\
\hline 51 & & & 67157 & & & & & & & 69 & 2 \\
\hline 2. & & & & & & & & & & & 2 \\
\hline 53. & & & & 12.59 & & & & & & & 2 \\
\hline 4. & & & +6549.6 & 9.81 & & & & & & & 2 \\
\hline 5 & & & & & & & & & & & 2 \\
\hline 6 & & & & & & & & & & & 2 \\
\hline & & & & & & & & & & & 2 \\
\hline & & & & & & & & & & & 2 \\
\hline & & & & & & & & & & & 2 \\
\hline 60. & & & & & & & & & & & 2 \\
\hline 1 & & & & & & & & & & & 2 \\
\hline & & & & & & & & & & & 2 \\
\hline 63. & & & & & & & & & & & 2 \\
\hline 4 & & & & & & & & & & & 2 \\
\hline & & & & & & & & & & & 2 \\
\hline & & & & & & & & & & & 2 \\
\hline & & & & & & & & & & & 2 \\
\hline & $+66^{\circ} 88 p$ & & & & & & & & & & 2 \\
\hline & & & $6=$ & & & & & & & & 2 \\
\hline 70. & & & & & & & & & & & 2 \\
\hline 71 & & & & & & & & & & & \\
\hline & & & & & & & & & & & 2 \\
\hline 73. & $+66^{\circ} 89$ & & & & & & & & & .86 & $?$ \\
\hline 74. & & & & & & & & & & & 2 \\
\hline 75. & & & & 10.33 & & & & & & & \\
\hline 76. & +6 & & & & & & & & & & 2 \\
\hline 77. & $+65^{\circ} 127$ & & +6629.4 & & & & & & & 0.32 & ? \\
\hline 78. & & & & & & & & & & 0.53 & \\
\hline & & & & & & & & & & 0.73 & \\
\hline 80. & & 10438 & & 11.47 & 2.83 & 2.08 & 1.35 & 0.70 & 0.26 & 0.55 & 2 \\
\hline
\end{tabular}


Table 1 (continued)

\begin{tabular}{|c|c|c|c|c|c|c|c|c|c|c|c|}
\hline No. & $\mathrm{BD}$ & $\begin{array}{l}\alpha(2000) \\
\mathrm{h}_{\mathrm{m}} \mathrm{s}\end{array}$ & $\delta(2000)$ & $V$ & $l \quad V^{\circ}$ & $P-V$ & $X-V$ & $Y-V$ & $Z-V$ & $V-S$ & $n$ \\
\hline 81. & & 10446 & +6703.1 & 11.33 & 2.55 & 2.05 & 1.47 & 0.62 & 0.25 & 0.61 & 2 \\
\hline 82 . & & 10503 & +6706.5 & 12.21 & 3.09 & 2.27 & 1.42 & 0.66 & 0.25 & 0.57 & 2 \\
\hline 83. & & 10509 & +6734.8 & 12.31 & 3.19 & 2.36 & 1.55 & 0.79 & 0.28 & 0.66 & 2 \\
\hline 84. & & 10534 & +6705.6 & 12.11 & 2.75 & 2.22 & 1.54 & 0.63 & 0.26 & 0.66 & 2 \\
\hline 85. & & 10538 & +6708.0 & 12.71 & & & 3.28: & 1.47 & 0.54 & 1.30 & 2 \\
\hline 86. & & 10540 & +6710.3 & 12.24 & 3.85 & 3.07 & $2.26:$ & 0.99 & 0.36 & 0.93 & 2 \\
\hline 87. & $+66^{\circ} 92$ & 10549 & +6718.7 & 10.24 & 2.36 & 1.86 & 1.33 & 0.58 & 0.23 & 0.57 & 2 \\
\hline 88. & $+65^{\circ} 129$ & 10553 & +6558.3 & 7.24 & 1.70 & 1.17 & 0.62 & 0.31 & 0.12 & 0.29 & 2 \\
\hline 89. & $+65^{\circ} 128$ & 10556 & +6553.7 & 9.35 & 2.12 & 1.44 & 0.69 & 0.32 & 0.11 & 0.27 & 2 \\
\hline 90. & & 10611 & +6704.1 & 10.78 & 2.99 & 2.17 & 1.25 & 0.63 & 0.22 & 0.49 & 2 \\
\hline 91. & & 10625 & +6632.7 & 10.98 & & & 4.09 & 1.86 & 0.70 & 1.64 & 2 \\
\hline 92. & $+65^{\circ} 130$ & 10626 & +6626.8 & 8.51 & 2.27 & 1.56 & 0.77 & 0.37 & 0.14 & 0.29 & 2 \\
\hline 93. & & 10657 & +6706.7 & 11.10 & & $4.52:$ & 3.20 & 1.27 & 0.54 & 1.13 & 2 \\
\hline 94. & & 10730 & +6709.2 & 12.06 & 2.86 & 2.21 & 1.57 & 0.72 & 0.26 & 0.62 & 2 \\
\hline 95. & $+66^{\circ} 93$ & $\begin{array}{lll}108 & 11\end{array}$ & +6718.1 & 10.44 & 2.40 & 1.78 & 1.13 & 0.50 & 0.20 & 0.45 & 2 \\
\hline 96. & $+66^{\circ} 94 b$ & 10814 & +6728.4 & 11.73 & 3.21 : & $2.45:$ & 1.70: & 0.78 & 0.26 & 0.70 & 1 \\
\hline 97. & $+66^{\circ} 94 \mathrm{a}$ & $\begin{array}{lll}108 & 17\end{array}$ & +6729.0 & 8.57 & 5.16 & 4.32 & 3.08 & 1.33 & 0.54 & 1.18 & 2 \\
\hline 98. & $+66^{\circ} 95$ & 10825 & +6648.9 & 10.03 & 2.84 & 2.35 & 1.60 & 0.63 & 0.25 & 0.61 & 2 \\
\hline 99 & $+65^{\circ} 131$ & 10840 & +6612.8 & 10.53 & 2.91 & 2.17: & 1.47 & 0.80 & 0.28 & 0.63 & 2 \\
\hline 100. & & 10858 & +6702.9 & 11.80 & 3.65 & $2.72:$ & 1.81 & 0.89 & 0.32 & 0.72 & 2 \\
\hline 101. & $+66^{\circ} 96$ & 10932 & +6742.7 & 10.88 & 2.38 & 1.83 & 1.24 & 0.54 & 0.22 & 0.50 & 1 \\
\hline 102. & $+65^{\circ} 132$ & 10942 & +6616.4 & 9.83 & 2.11 & 1.54 & 0.88 & 0.44 & 0.16 & 0.34 & 2 \\
\hline 103. & & 10945 & +6651.3 & 11.74 & 2.46 & 1.94 & 1.36 & 0.61 & 0.23 & 0.57 & 2 \\
\hline 104. & $+65^{\circ} 133$ & 11004 & +6546.4 & 10.81 & 2.36 & 1.71 & 0.83 & 0.40 & 0.15 & 0.28 & 1 \\
\hline 105. & $+65^{\circ} 134$ & 11024 & +6608.0 & 8.85 & 3.91 & 3.33 & 2.36 & 0.94 & 0.37 & 0.83 & 2 \\
\hline 106. & $+65^{\circ} 135$ & 11023 & +6546.4 & 9.08 & 2.33 & 1.69 & 1.05 & 0.46 & 0.17 & 0.42 & 2 \\
\hline 107 & $+65^{\circ} 136$ & 11031 & +6644.5 & 9.11 & 2.45 & 1.96 & 1.36 & 0.55 & 0.22 & 0.55 & 2 \\
\hline 108. & $+65^{\circ} 137$ & 11049 & +6549.0 & 10.48 & 2.39 & 1.75 & 1.04 & 0.46 & 0.17 & 0.41 & 2 \\
\hline 109. & $+65^{\circ} 138$ & 11126 & +6624.0 & 10.45 & 2.67 & 2.12 & 1.48 & 0.64 & 0.26 & 0.58 & 2 \\
\hline 110. & $+65^{\circ} 139$ & 11130 & +6603.5 & 9.72 & 2.31 & 1.67 & 0.94 & 0.38 & 0.14 & 0.37 & 2 \\
\hline 111. & & 11139 & +6703.3 & 11.48 & 3.31 & 2.43 & 1.62 & 0.83 & 0.27 & 0.66 & 2 \\
\hline 112. & $+65^{\circ} 140$ & 11149 & $\begin{array}{r}6552.4 \\
\end{array}$ & 8.50 & 4.22 & 3.57 & 2.43 & 0.91 & 0.38 & 0.86 & 2 \\
\hline 113. & $+65^{\circ} 141$ & 11227 & +6625.6 & 10.19 & 4.50 & $3.78:$ & 2.62 & 1.05 & 0.42 & 0.94 & 2 \\
\hline 114. & $+65^{\circ} 142$ & 11240 & +6609.8 & 9.49 & 2.95 & 2.10 & 1.37 & 0.73 & 0.25 & 0.57 & 2 \\
\hline 115. & $+66^{\circ} 97$ & 11301 & +6738.1 & 9.74 & 2.44 & 1.79 & 1.05 & 0.43 & 0.18 & 0.36 & 2 \\
\hline 116. & $+65^{\circ} 143$ & 11425 & +6643.3 & 10.60 & 2.39 & 1.87 & 1.32 & 0.54 & 0.21 & 0.52 & 2 \\
\hline 117. & $+65^{\circ} 144$ & 11428 & +6547.1 & 8.94 & 4.08 & 3.41 & 2.36 & 0.91 & 0.38 & 0.83 & 2 \\
\hline 118. & $+66^{\circ} 99$ & 11501 & +6719.0 & 10.38 & 2.40 & 1.87 & 1.31 & 0.56 & 0.22 & 0.54 & 2 \\
\hline 119. & $+66^{\circ} 98$ & 11503 & +6714.2 & 10.12 & 2.29 & 1.71 & 1.10 & 0.49 & 0.19 & 0.45 & 2 \\
\hline 120. & $+65^{\circ} 145$ & 11612 & +6607.8 & 8.57 & 2.24 & 1.65 & 1.03 & 0.44 & 0.17 & 0.41 & 2 \\
\hline
\end{tabular}


Table 1 (continued)

\begin{tabular}{|c|c|c|c|c|c|c|c|c|c|c|}
\hline $\mathrm{BD}$ & $\begin{array}{l}\alpha(2000) \\
\text { h m s }\end{array}$ & $\delta(2000)$ & $V$ & $U-V$ & $P-V$ & $X-V$ & $Y-V$ & $Z-V$ & $V-S$ & $n$ \\
\hline 121. $+66^{\circ} 100$ & 11645 & +6730.1 & 10.46 & 3.15 & 2.33 & 1.62 & 0.83 & 0.29 & 0.69 & 2 \\
\hline 122. $+66^{\circ} 101$ & 11700 & +6733.0 & 10.91 & 2.41 & 1.95 & 1.37 & 0.55 & 0.21 & 0.55 & 2 \\
\hline $123 .+65^{\circ} 146$ & 11723 & +6557.9 & 10.25 & 4.31 & 3.62 & 2.52 & 1.01 & 0.41 & 0.90 & 2 \\
\hline 124. $+66^{\circ} 102$ & 11736 & +6742.9 & 9.86 & 2.21 & 1.65 & 1.02 & 0.43 & 0.16 & 0.40 & 2 \\
\hline 125. $+65^{\circ} 147$ & 11747 & +6549.7 & 9.66 & 2.43 & 1.78 & 1.14 & 0.51 & 0.19 & 0.46 & 2 \\
\hline 126. $+65^{\circ} 148$ & 11757 & +6549.9 & 10.32 & 2.48 & 1.83 & 1.08 & 0.46 & 0.17 & 0.43 & 2 \\
\hline 127. $+65^{\circ} 149$ & 11836 & +6621.0 & 10.62 & 2.39 & 1.77 & 1.11 & 0.50 & 0.20 & 0.42 & 2 \\
\hline $128 .+65^{\circ} 150$ & 11840 & +6606.4 & 11.44 & 2.51 & 1.91 & 1.23 & 0.53 & 0.20 & 0.48 & 1 \\
\hline $129 .+65^{\circ} 152$ & 11938 & +6631.4 & 11.07 & 2.29 & 1.75 & 1.21 & 0.53 & 0.19 & 0.49 & 2 \\
\hline 130. $+66^{\circ} 103$ & 11948 & +6712.1 & 9.22 & 2.13 & 1.54 & 0.77 & 0.32 & 0.12 & 0.24 & 2 \\
\hline 131. $+66^{\circ} 104$ & & +6726.8 & 10.53 & 2.66 & 2.00 & & & & 0.51 & 2 \\
\hline 132. $+66^{\circ} 105$ & 12037 & +6714.0 & 8.44 & 2.29 & 1.67 & 0.97 & 0.39 & 0.15 & 0.35 & 2 \\
\hline $133 .+65^{\circ} 153$ & 12050 & +6547.4 & 9.78 & 2.68 & 1.97 & 1.24 & 0.55 & 0.21 & 0.50 & 2 \\
\hline 134. $+66^{\circ} 106$ & 12054 & +6741.9 & 9.41 & 2.44 & 1.91 & 1.31 & 0.55 & 0.21 & 0.53 & 2 \\
\hline 135. $+66^{\circ} 107$ & 12141 & +6714.0 & 10.22 & 2.30 & 1.76 & 1.18 & 0.50 & 0.19 & 0.47 & 2 \\
\hline 136. $+66^{\circ} 108 \mathrm{a}$ & 12144 & +6719.6 & 10.89 & 2.64 & 2.11 & 1.46 & 0.60 & 0.22 & 0.58 & 2 \\
\hline 137. $+66^{\circ} 108 b$ & 12145 & +6719.1 & 11.75 & 2.62 & 2.11 & 1.45 & 0.58 & 0.22 : & 0.59 & 2 \\
\hline 138. $+65^{\circ} 154$ & 12151 & +6558.3 & 10.61 & 2.64 & 1.92 & 1.11 & 0.55 & 0.18 & 0.41 & 2 \\
\hline 139. $+66^{\circ} 109$ & 12219 & +6729.2 & 10.62 & 2.79 & 2.01 & 1.15 & 0.57 & 0.21 & 0.45 & 1 \\
\hline $140 .+65^{\circ} 155$ & 12229 & +6606.4 & 8.81 & 5.58 & 4.73 & 3.28 & 1.23 & 0.58 & 1.13 & 2 \\
\hline 141. $+65^{\circ} 156$ & & & & 2.29 & 1.74 & & 0.52 & 0.20 & 0.50 & 2 \\
\hline $142 .+65^{\circ} 157$ & 12343 & +6620.5 & 10.47 & 2.36 & 1.84 & 1.24 & 0.52 & 0.20 & 0.51 & 1 \\
\hline 143. $+65^{\circ} 158$ & 12355 & +6606.2 & 10.06 & 2.44 & 1.84 & 1.24 & 0.54 & 0.20 & 0.50 & 2 \\
\hline 144. $+66^{\circ} 111$ & 12402 & +6726.9 & 9.15 & 2.62 & 1.85 & 1.14 & 0.60 & 0.21 & 0.48 & 1 \\
\hline 145. $+66^{\circ} 112$ & 12403 & +6708.9 & 10.19 & & & 4.16: & 1.67 & 0.69 & 1.50 & 1 \\
\hline 146. $+65^{\circ} 159$ & 12413 & +6621.6 & 10.70 & 2.26 & 1.76 & 1.22 & 0.56 & 0.21 & 0.48 & 1 \\
\hline 147. $+66^{\circ} 114$ & 12649 & +6704.1 & 8.77 & 2.29 & 1.64 & 0.94 & 0.39 & 0.15 & 0.36 & 1 \\
\hline 148. $+66^{\circ} 113$ & 12650 & +6713.4 & 10.55 & 2.11 & 1.61 & 1.04 & 0.46 & 0.18 & 0.42 & 1 \\
\hline 149. $+66^{\circ} 116 \mathrm{p}$ & 12705 & +6657.7 & 9.89 & 2.91 & 2.00 & 1.13 & 0.56 & 0.21 & 0.46 & 1 \\
\hline$+66^{\circ} 116$ & 12706 & +6658.5 & 9.28 & 2.56 & 1.81 & 1.09 & 0.57 & 0.21 & 0.44 & 1 \\
\hline 151. $+66^{\circ} 118$ & 12757 & +6707.0 & 9.45 & & 4.96: & 3.42: & 1.28 & 0.58 & 1.21 & 1 \\
\hline 152. $+66^{\circ} 119$ & 12814 & +6710.3 & 8.92 & 2.21 & 1.67 & 1.09 & 0.55 & 0.19 & 0.46 & 1 \\
\hline
\end{tabular}


To obtain the interstellar monochromatic extinction $A_{V}$ at the wavelength $\lambda=544 \mathrm{~nm}$, which is the effective wavelength of the Vilnius system's $V$ passband, the ratio $R_{Y V}=A_{V} / E_{Y-V}$ has been used. The ratio $R_{Y V}$ depends slightly on the spectral type and the luminosity of star. Table 2 gives the values of $R_{Y V}$ for various spectral types and luminosities. These values were obtained by calculating the synthetic color excesses $E_{Y-V}$ for the amount of interstellar matter $x=1\left(E_{B-V}=1\right)$ from energy distribution of normal stars (Sviderskiene 1988), response functions of the Vilnius system and the mean extinction law, normalized to $R_{B V}=3.15$, given by Straižys (1992). It is evident that the mean value $R_{Y V}=4.15$ is good approximation for all spectral types and luminosities excluding only very late $\mathrm{M}$-type stars.

Table 2. The ratios of interstellar extinction $A_{V}$ to color excess $E_{Y-V}$.

\begin{tabular}{rl|l|rl|l}
\hline \multicolumn{2}{c|}{ Sp } & $R_{Y V}$ & Sp & $R_{Y V}$ \\
\hline B0-A5 & V-III & 4.14 & K0 & III & 4.17 \\
A7-F8 & V-III & 4.15 & K2-M1 & III & 4.18 \\
G0-G5 & V-III & 4.16 & M2-M3 & III & 4.19 \\
K0 & V & 4.16 & M4 & III & 4.20 \\
K2 & V & 4.15 & M5 & III & 4.25 \\
K3 & V & 4.14 & M7 & III & 4.29 \\
K4 & V & 4.13 & B0-F0 & I & 4.15 \\
K5-M0 & V & 4.11 & F5 & I & 4.16 \\
M2 & V & 4.13 & F8-G5 & I & 4.17 \\
M3 & V & 4.14 & G8 & I & 4.19 \\
M5 & V & 4.16 & K2-K4 & I & 4.20 \\
M7 & V & 4.22 & M2 & I & 4.26 \\
\hline
\end{tabular}

For a comparison with the $B V$ system, in Table 3 we give color excesses $E_{B-V}$, calculated with the relations given by Kuriliene \& Sūdžius (1974). The distance was found by the equation

$$
\log r=\frac{V-M_{V}+5-A_{V}}{5} .
$$


The results of photometric classification, interstellar extinction and distance determination are given in Table 3, which contains: identification number, HD number, spectral class from the PPM Star Catalogue (1992), photometric spectral type, $M_{V}, E_{Y-V}, E_{B-V}$, $A_{V}$, distance $r$ in parsecs, rounded to the nearest number multiple to 10 for $r>150 \mathrm{pc}$, and the weight of the two-dimensional quantification.

The maximum expected errors of $A_{V}$ do not exceed $0.15 \mathrm{mag}$, the typical errors of absolute magnitudes $M_{V}$ are \pm 0.5 mag.

\section{INTERSTELLAR EXTINCTION IN THE AREA}

Fig. 2 shows the dependence of extinction on distance for the investigated area, plotted from the data of Table 3 . Two regions with different run of extinction with distance may be separated: one of them is the Dark Cloud shown in Fig. 1 and the second one is more transparent region surrounding the cloud. The northern boundary of the Dark Cloud is not well defined due to small number of stars observed in this area. The slightly reddened stars start to appear at a distance of $160 \mathrm{pc}$ in both regions. In the Dark Cloud region at $r$ $=250 \mathrm{pc}$, the interstellar extinction begins to grow up very fast; in the surrounding region it starts to grow only at $\sim 400 \mathrm{pc}$. A possible explanation is that we have here two dark clouds: the more distant cloud covers both regions and the nearer one covers only the Dark Cloud region. The inclined rise of extinction with distance in the Dark Cloud region may indicate that the ratio $R_{Y V}$ is larger than the normal value of 4.15. To get the vertical rise of extinction at $r$ $=400 \mathrm{pc}$, this ratio should be of the order of 6 (or $R_{B V} \approx 4.5$ ).

Several stars from the Dark Cloud region (Nos. 42, 57, 70, 71, $113,136,137)$ show the extinction run with distance, which is more typical for the surrounding field. It may be caused either by the absolute magnitude errors or by transparent windows in the dust cloud. The presence of such windows is confirmed by star counts in small areas where these stars were observed. Three stars, i.e. No. 42 (K0 II, $\left.M_{V}=-2.0\right)$, No. $57\left(\mathrm{BD}+66^{\circ} 86, \mathrm{G} 4 \mathrm{II}, M_{V}=-3.9\right)$ and No. $79\left(\mathrm{BD}+66^{\circ} 91, \mathrm{~B} 2\right.$ II, $\left.M_{V}=-4.7\right)$ seem to be situated behind the dark cloud complex, but their absolute magnitudes are not very reliable. There is no indication at which distance thedark cloud ends. If the cloud extends to $r=1 \mathrm{kpc}$, as Fig. 2 seems to indicate, then it should be rather high (about $100 \mathrm{pc}$ ) above the galactic plane. To verify, more distant stars should be measured. 


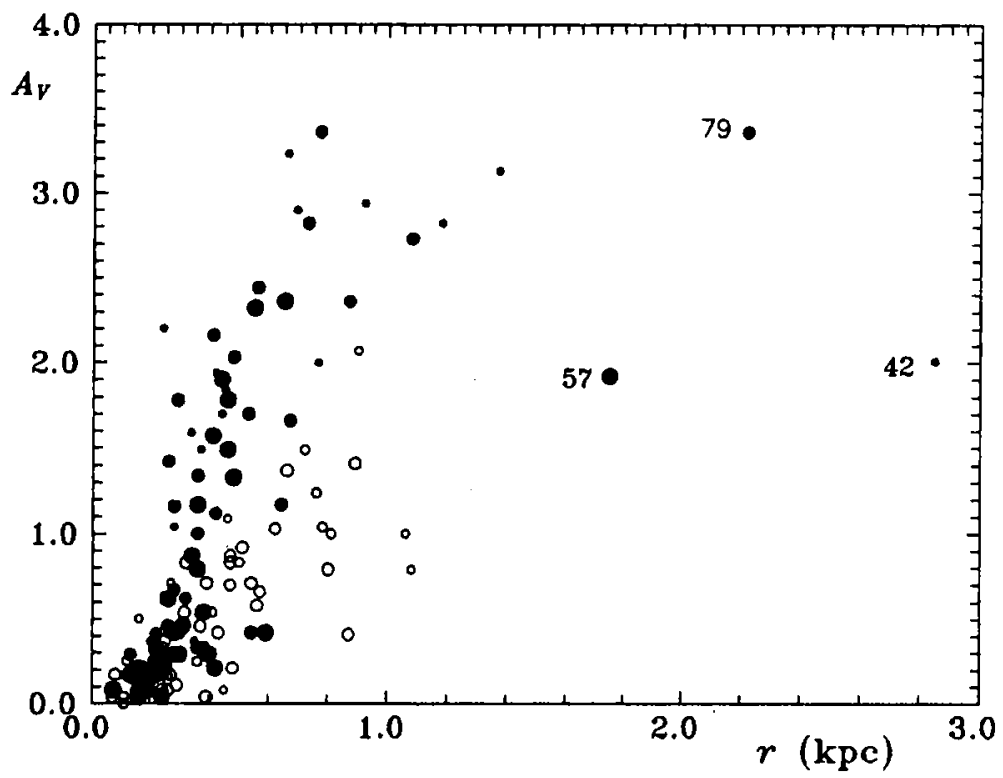

Fig. 2. Dependence of interstellar extinction on distance in the investigated area. Dots are stars situated in the Dark Cloud area, circles are for more transparent area. Different sizes of dots and circles correspond to different accuracy of classification.

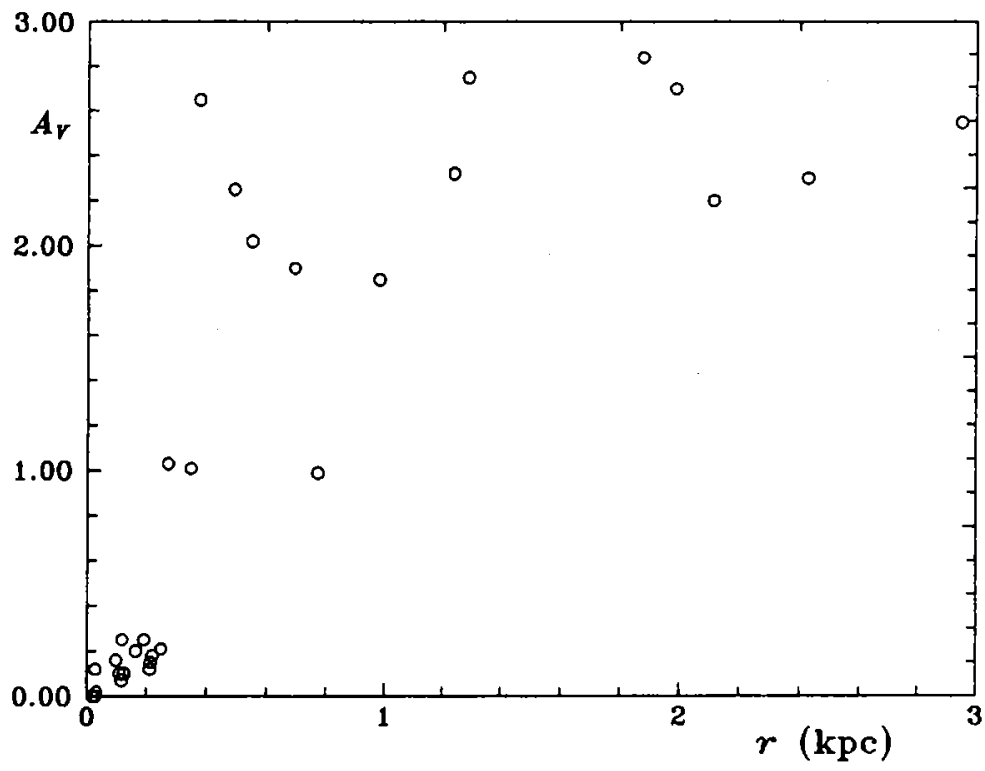

Fig. 3. Dependence of interstellar extinction on distance in the galactic belt with the coordinates $l=120^{\circ}-130^{\circ}, b=+2^{\circ}-+10^{\circ}$. 
Table 3. Results of quantification, extinction and distance determination.

\begin{tabular}{|c|c|c|c|c|c|c|c|c|c|}
\hline No. & HD & $\begin{array}{c}\text { PPM } \\
\text { sp. type }\end{array}$ & $\begin{array}{l}\text { Photom. } \\
\text { sp. type }\end{array}$ & $M_{V}$ & $E_{Y-V}$ & $E_{B-V}$ & $A_{V}$ & $\begin{array}{c}r \\
\mathrm{pc}\end{array}$ & $W$ \\
\hline $1 . *$ & & & K0 IV? & 3.4: & & & & & \\
\hline 2. & 4625 & G5 & F2 III & 1.4 & 0.02 & 0.03 & 0.08 & 450 & 1 \\
\hline 3 . & & $\mathrm{K} 2$ & K4 III & -1.7 & 0.48 & 0.55 & 2.17 & 740 & 1 \\
\hline 4. & & K0 & K3 III & 0.1 & 0.32 & 0.36 & 1.34 & 360 & 2 \\
\hline 5. & & & F5 V & 3.7 & 0.03 & 0.04 & 0.12 & 170 & 3 \\
\hline 6. & & $\mathrm{~A} 0$ & A0 III & -1.6 & 0.34 & 0.44 & 1.41 & 890 & 3 \\
\hline 7. & & & F4 IV & 3.0 & 0.04 & 0.05 & 0.17 & 240 & 3 \\
\hline 8. & 4829 & K0 & K2 III & 0.7 & 0.04 & 0.05 & 0.17 & 270 & 3 \\
\hline 9. & 4828 & $\mathrm{~K} 5$ & M0 III & -0.6 & 0.38 & 0.42 & 1.59 & 340 & 1 \\
\hline 10. & & A5 & F0 IV & 2.2 & 0.03 & 0.03 & 0.11 & 290 & 3 \\
\hline 11. & 4948 & $\mathrm{~A} 2$ & $\mathrm{Al} \mathrm{V}$ & 1.5 & 0.05 & 0.07 & 0.21 & 210 & 3 \\
\hline 12. & & B5 & B3 V & -1.9 & 0.50 & 0.66 & 2.07 & 900 & 1 \\
\hline 13. & & & K0 III & -0.6 & 0.24 & 0.29 & 1.00 & 1060 & 1 \\
\hline 14. & & & $\mathrm{G} 1 \mathrm{~V}$ & 4.8 & 0.01 & 0.02 & 0.04 & 110 & 2 \\
\hline 15. & & & F3 III & -0.4 & 0.17 & 0.22 & 0.71 & 540 & 3 \\
\hline 16. & 5110 & Fo & F2 IV & 2.9 & 0.02 & 0.03 & 0.08 & 65 & 3 \\
\hline 17. & & & F8 IV & 2.7 & 0.07 & 0.09 & 0.29 & 260 & 3 \\
\hline 18. & 5162 & G5 & F8 V & 4.7 & 0.02 & 0.03 & 0.08 & 71 & 3 \\
\hline 19. & 5257 & F5 & F3 IV & 2.9 & 0.05 & 0.07 & 0.21 & 180 & 3 \\
\hline 20. & & & $\mathrm{~F} 6 \mathrm{~V}$ & 3.7 & 0.12 & 0.16 & 0.50 & 170 & 2 \\
\hline 21. & & F8 & F2 III & 1.4 & 0.17 & 0.22 & 0.71 & 390 & 3 \\
\hline 22. & & & K3 III & 0.4 & 0.19 & 0.22 & 0.79 & 1080 & 1 \\
\hline 23 . & & G0 & F8 IV & 3.0 & 0.05 & 0.06 & 0.21 & 200 & 3 \\
\hline 24. & & B8 & B9 III & -1.1 & 0.49 & 0.64 & 2.03 & 480 & 2 \\
\hline 25 . & 5326 & AO & B4 V & -0.9 & 0.21 & 0.28 & 0.87 & 470 & 3 \\
\hline 26. & & F8 & G1 III & 0.9 & 0.41 & 0.51 & 1.71 & 910 & 1 \\
\hline 27. & 5352 & A2 & Al III & -0.9 & 0.25 & 0.32 & 1.03 & 620 & 3 \\
\hline 28. & & $\mathrm{KO}$ & KO III & 0.0 & 0.22 & 0.26 & 0.92 & 510 & 3 \\
\hline 29. & & & G5 III & 0.7 & 0.38 & 0.46 & 1.59 & 400 & 1 \\
\hline 30. & 5476 & F5 & F1 IV & 2.0 & 0.04 & 0.06 & 0.17 & 240 & 2 \\
\hline 31 .* & & M0 & M1 III & -1.6 & 0.44 & 0.49 & 1.84 & 450 & 1 \\
\hline 32 . & & F8 & F5 V & 3.6 & 0.04 & 0.05 & 0.17 & 170 & 3 \\
\hline 33. & & A5 & A8 III & $\begin{array}{l}0.0 \\
1.5\end{array}$ & 0.08 & 0.10 & 0.33 & 370 & 3 \\
\hline 34. & 5535 & A0 & $\mathrm{A} 1 \mathrm{~V}$ & 1.3 & 0.20 & 0.26 & 0.83 & 320 & 3 \\
\hline 35 . & 5550 & $\mathrm{~B} 9$ & B9 V & 1.3 & 0.04 & 0.05 & 0.17 & 80 & 3 \\
\hline 36. & & A5 & F0 III & 1.5 & 0.05 & 0.07 & 0.21 & 480 & 3 \\
\hline 37. & 5563 & F2 & F2 IV & 2.5 & 0.04 & 0.04 & 0.17 & 140 & 3 \\
\hline 38. & & & F1 III & 0.8 & 0.30 & 0.38 & 1.24 & 760 & 2 \\
\hline 39. & & & G8 IV & 2.0 & 0.34 & 0.42 & 1.42 & 260 & 2 \\
\hline 40. & & & F8 V & 4.1 & 0.05 & 0.06 & 0.21 & 210 & 3 \\
\hline 41. & & & F9 V & 4.3 & 0.15 & 0.19 & 0.62 & 320 & 2 \\
\hline 42. & & & K0 II & -2.0 & 0.48 & 0.56 & 2.01 & 2850 & 1 \\
\hline 43. & & Mo & M2 III & -0.5 & 0.26 & 0.29 & 1.09 & 460 & 1 \\
\hline 44. & & & F1 III & -0.1 & 0.36 & 0.46 & 1.49 & 720 & 2 \\
\hline 45. & & F5 & $\mathrm{F} 2 \mathrm{~V}$ & 3.0 & 0.04 & 0.05 & 0.17 & 220 & 3 \\
\hline
\end{tabular}


Table 3 (continued)

\begin{tabular}{|c|c|c|c|c|c|c|c|c|c|}
\hline No. & HD & $\begin{array}{c}\text { PPM } \\
\text { sp. type }\end{array}$ & $\begin{array}{l}\text { Photom. } \\
\text { sp. type }\end{array}$ & $M_{V}$ & $E_{Y-V}$ & $E_{B-V}$ & $A_{V}$ & $\begin{array}{c}r \\
\mathrm{pc}\end{array}$ & $W$ \\
\hline 46. & & & A5 V & 1.8 & 0.27 & 0.35 & 1.12 & 420 & 2 \\
\hline 47. & & & G7 III & 1.0 & 0.28 & 0.34 & 1.17 & 360 & 3 \\
\hline 48 & & & F5 V & 3.6 & 0.07 & 0.09 & 0.29 & 300 & 3 \\
\hline 49. & & & K1 III & -0.4 & 0.19 & 0.23 & 0.79 & 800 & 3 \\
\hline 50. & & & K1 IV & 3.7 & 0.25 & 0.30 & 1.04 & 280 & 1 \\
\hline 51. & & & F6 V & 3.8 & 0.15 & 0.20 & 0.62 & 260 & 3 \\
\hline 52. & & & K1 III & 1.1 & 0.10 & 0.12 & 0.42 & 430 & 3 \\
\hline 53. & & & $\mathrm{G} 2 \mathrm{~V}$ & 4.5 & 0.09 & 0.12 & 0.37 & 350 & 1 \\
\hline 54. & & $\mathrm{~K} 2$ & K0 III & -0.7 & 0.25 & 0.30 & 1.04 & 780 & 2 \\
\hline 55. & & $\mathrm{~A} 0$ & $\mathrm{~A} 3 \mathrm{~V}$ & 1.5 & 0.28 & 0.36 & 1.16 & 280 & 2 \\
\hline 56. & & & B9 III & -1.7 & 0.78 & 1.02 & 3.23 & 660 & 1 \\
\hline 57. & & $\mathrm{~K} 2$ & G4 II & -3.9 & 0.46 & 0.56 & 1.92 & 1750 & 3 \\
\hline 58. & 5849 & B9 & B6 IV & -1.0 & 0.33 & 0.43 & 1.37 & 660 & 3 \\
\hline 59. & & & F5 V & 3.6 & 0.01 & 0.02 & 0.04 & 240 & 3 \\
\hline 60. & 5889 & G0 & F8 V & 4.1 & 0.01 & 0.01 & 0.04 & 72 & 3 \\
\hline 61. & & & $\mathrm{~K} 2 \mathrm{~V}$ & 5.8 & 0.07 & 0.08 & 0.29 & 130 & 2 \\
\hline 62. & & KO & K1 IV & 4.4 & 0.00 & 0.00 & 0.00 & 110 & 2 \\
\hline 63. & & & F4 V & 3.3 & 0.11 & 0.14 & 0.46 & 310 & 3 \\
\hline 64. & & & F3 V & 3.4 & 0.43 & 0.54 & 1.78 & 300 & 2 \\
\hline 65. & & & $\mathrm{~A} 0 \mathrm{~V}$ & 1.2 & 0.36 & 0.48 & 1.49 & 460 & 3 \\
\hline 66. & & & F3 V & 3.3 & 0.07 & 0.09 & 0.29 & 270 & 3 \\
\hline 67. & & & K1 III & 1.4 & 0.20 & 0.23 & 0.83 & 470 & 3 \\
\hline 68. & & F5 & F6 V & 4.1 & 0.05 & 0.06 & 0.21 & 160 & 3 \\
\hline 69. & & & G0 III & 1.7 & 0.10 & 0.12 & 0.42 & 590 & 3 \\
\hline 70. & & & $\mathrm{~A} 0 \mathrm{~V}$ & 0.6 & 0.57 & 0.75 & 2.36 & 650 & 3 \\
\hline 71. & & $\mathrm{~K} 2$ & K2 III & 0.9 & 0.10 & 0.12 & 0.42 & 540 & 2 \\
\hline 72. & & F5 & F $4 \mathrm{~V}$ & 3.3 & 0.12 & 0.15 & 0.50 & 160 & 1 \\
\hline 73. & & G5 & F1 II & -4.0 & 0.68 & 0.87 & 2.82 & 1180 & 1 \\
\hline 74. & & & $\mathrm{~A} 5 \mathrm{~V}$ & 1.8 & 0.38 & 0.49 & 1.57 & 410 & 3 \\
\hline 75 . & & & F5 IV & 3.0 & 0.09 & 0.11 & 0.37 & 250 & 3 \\
\hline 76. & 6091 & F8 & F4 V & 3.2 & 0.01 & 0.02 & 0.04 & 160 & 3 \\
\hline 77. & & & A5 IV & 1.4 & 0.17 & 0.21 & 0.70 & 470 & 3 \\
\hline 78. & & & F5 V & 3.6 & 0.10 & 0.12 & 0.41 & 260 & 1 \\
\hline 79. & & & B2 I & -4.7 & 0.81 & 1.09 & 3.36 & 2220 & 2 \\
\hline 80. & & & B8 III & -0.6 & 0.57 & 0.75 & 2.36 & 870 & 2 \\
\hline 81. & & & G0 V & 4.4 & 0.09 & 0.11 & 0.37 & 210 & 1 \\
\hline 82. & & & $\mathrm{~A} 8 \mathrm{~V}$ & 2.9 & 0.36 & 0.47 & 1.49 & 370 & 1 \\
\hline 83. & & & B8 III & -0.6 & 0.66 & 0.87 & 2.73 & 1080 & 2 \\
\hline 84. & & & G3 V & 4.8 & 0.07 & 0.08 & 0.29 & 250 & 1 \\
\hline 85. & & & G8 III & -1.1 & 0.75 & 0.91 & 3.13 & 1370 & $\overrightarrow{1}$ \\
\hline 86. & & & F5 IV & 3.1 & 0.53 & 0.68 & 2.20 & 240 & 1 \\
\hline 87. & & & F8 V & 4.2 & 0.07 & 0.08 & 0.29 & 140 & 1 \\
\hline $88 .^{*}$ & 6343 & B8 & B7 III & -1.3 & 0.19 & 0.26 & 0.79 & 360 & 3 \\
\hline 89. & & $\mathrm{~A}$ & AO III & -0.1 & 0.16 & 0.21 & 0.66 & 570 & 3 \\
\hline 90. & & & $\mathrm{~A} 0 \mathrm{~V}$ & 0.7 & 0.47 & 0.61 & 1.94 & 420 & 1 \\
\hline
\end{tabular}


Table 3 (continued)

\begin{tabular}{|c|c|c|c|c|c|c|c|c|c|}
\hline No. & HD & $\begin{array}{c}\text { PPM } \\
\text { sp. type }\end{array}$ & $\begin{array}{l}\text { Photom. } \\
\text { sp. type }\end{array}$ & $M_{V}$ & $E_{Y-V}$ & $E_{B-V}$ & $A_{V}$ & $\begin{array}{r}r \\
\mathrm{pc}\end{array}$ & $W$ \\
\hline $91 . *$ & & & $\mathrm{~K}-\mathrm{M}$ ? & & & & & & \\
\hline $\begin{array}{l}92 . \\
93 . *\end{array}$ & 6415 & $\mathrm{~A} 0$ & $\begin{array}{l}\text { A0 IIl } \\
\mathrm{K}-\mathrm{M} ?\end{array}$ & 0.0 & 0.21 & 0.27 & 0.87 & 340 & 3 \\
\hline 94. & & & F6 IV & 3.3 & 0.24 & 0.30 & 1.00 & 350 & 2 \\
\hline 95. & & F0 & F3 IV & 2.3 & 0.08 & 0.10 & 0.33 & 360 & 2 \\
\hline $96 . *$ & & & F7 III & 1.7 & 0.29 & 0.37 & 1.20 & 580 & 1 \\
\hline $97 . *$ & 6599 & K0 & K0 III & -0.5 & 0.56 & 0.67 & 2.34 & 220 & 1 \\
\hline 98. & & & G5 IV & 3.4 & 0.04 & 0.05 & 0.17 & 200 & 3 \\
\hline 99. & & & B7 III & -1.6 & 0.68 & 0.89 & 2.82 & 730 & 2 \\
\hline 100. & & & F1 III & 1.6 & 0.52 & 0.66 & 2.16 & 410 & 2 \\
\hline 101. & & & F5 V & 3.6 & 0.08 & 0.11 & 0.33 & 250 & 3 \\
\hline 102. & & F8 & $\mathrm{B} 8 \mathrm{~V}$ & 0.1 & 0.32 & 0.43 & 1.33 & 480 & 3 \\
\hline 103. & & & F8 V & 4.1 & 0.10 & 0.12 & 0.42 & 280 & 3 \\
\hline 104.* & & & $\mathrm{A} 1 \mathrm{~V}$ & 1.5 & 0.20 & 0.27 & 0.83 & 500 & 2 \\
\hline 105. & 6821 & Ko & K0 III & 1.0 & 0.17 & 0.20 & 0.71 & 270 & 1 \\
\hline 106. & 6831 & F2 & F3 III & 1.8 & 0.04 & 0.05 & 0.17 & 260 & 2 \\
\hline 107. & 6830 & G0 & Go V & 4.5 & 0.02 & 0.03 & 0.08 & 80 & 2 \\
\hline 108. & & & F1 IV & 2.2 & 0.11 & 0.15 & 0.46 & 370 & 3 \\
\hline 109. & & & G0 IV & 2.9 & 0.11 & 0.13 & 0.46 & 260 & 2 \\
\hline 110. & & A5 & F1 III & 1.7 & 0.01 & 0.01 & 0.04 & 390 & 3 \\
\hline $111 .^{*}$ & & & B8 III & -0.6 & 0.70 & 0.92 & 2.90 & 690 & 1 \\
\hline 112. & 6985 & K0 & K1 III & 0.7 & 0.10 & 0.11 & 0.42 & 300 & 3 \\
\hline 113. & & G5 & K0 III & 0.0 & 0.28 & 0.34 & 1.17 & 640 & 2 \\
\hline 114. & & B5 & B9 III & -1.7 & 0.59 & 0.78 & 2.44 & 560 & 2 \\
\hline 115. & & A5 & F2 III & 1.4 & 0.02 & 0.03 & 0.08 & 450 & 1 \\
\hline 116. & & & F8 V & 4.2 & 0.03 & 0.04 & 0.12 & 180 & 1 \\
\hline 117. & 7263 & K0 & K0 III & -0.4 & 0.14 & 0.17 & 0.58 & 560 & 3 \\
\hline 118. & & & F8 V & 4.2 & 0.05 & 0.06 & 0.21 & 160 & 2 \\
\hline 119. & & Fo & F4 IV & 2.9 & 0.05 & 0.06 & 0.21 & 250 & 3 \\
\hline 120 & 7443 & F5 & F3 IV & 2.5 & 0.02 & 0.02 & 0.08 & 160 & 3 \\
\hline $121 .^{*}$ & & & B7 III & -2.3 & 0.71 & 0.94 & 2.94 & 920 & 1 \\
\hline 122. & & & G1 V & 4.7 & 0.01 & 0.02 & 0.04 & 170 & 2 \\
\hline 123. & & & K0 III & -0.3 & 0.24 & 0.29 & 1.00 & 810 & 2 \\
\hline 124. & & & F2 V & 2.9 & 0.03 & 0.03 & 0.12 & 230 & 3 \\
\hline 125. & & F5 & F5 III & 1.6 & 0.06 & 0.07 & 0.25 & 360 & 2 \\
\hline 126. & & & F0 IV & 2.3 & 0.13 & 0.17 & 0.54 & 320 & 3 \\
\hline 127. & & & F3 IV & 2.4 & 0.08 & 0.10 & 0.33 & 380 & 2 \\
\hline 128. & & & F2 V & 3.0 & 0.13 & 0.17 & 0.54 & 380 & 3 \\
\hline 129. & & & F5 V & 3.6 & 0.07 & 0.09 & 0.29 & 270 & 1 \\
\hline $130{ }^{*}$ & 7842 & $\mathrm{~A} 0$ & A5 V & 2.3 & 0.06 & 0.07 & 0.25 & 220 & 3 \\
\hline 131. & & & B7 IV & -0.5 & 0.56 & 0.74 & 2.32 & 550 & 3 \\
\hline 132. & 7901 & A3 & F1 III & 2.0 & 0.02 & 0.02 & 0.08 & 190 & 3 \\
\hline 133. & & & F3 III & 1.2 & 0.13 & 0.17 & 0.54 & 410 & 2 \\
\hline 134. & 7940 & K0 & F8 V & 3.7 & 0.04 & 0.05 & 0.17 & 130 & 3 \\
\hline 135. & & & F5 V & 3.6 & 0.04 & 0.05 & 0.17 & 190 & 3 \\
\hline
\end{tabular}


Table 3 (continued)

\begin{tabular}{llllllllll}
\hline No. & HD & $\begin{array}{c}\text { PPM } \\
\text { sp. type }\end{array}$ & $\begin{array}{l}\text { Photom. } \\
\text { sp. type }\end{array}$ & $M_{V}$ & $E_{Y-V}$ & $E_{B-V}$ & $A_{V}$ & $\begin{array}{r}r \\
\text { pc }\end{array}$ & $W$ \\
\hline 136. & & & G0 IV & 2.6 & 0.07 & 0.09 & 0.29 & 400 & 3 \\
137. & & & G1 IV & 3.4 & 0.05 & 0.06 & 0.21 & 420 & 3 \\
138. & & & B9 V & 0.7 & 0.41 & 0.54 & 1.70 & 440 & 1 \\
139. & & & A0 IV & 0.3 & 0.41 & 0.54 & 1.70 & 530 & 2 \\
140. & 8123 & K2 & K5 III & 0.9 & 0.16 & 0.18 & 0.67 & 280 & 2 \\
141. & & F8 & F5 V & 3.7 & 0.06 & 0.08 & 0.25 & 120 & 2 \\
142. & & & F7 V & 3.9 & 0.03 & 0.04 & 0.12 & 190 & 3 \\
$143 . *$ & & & F5 IV & 3.0 & 0.08 & 0.11 & 0.33 & 220 & 3 \\
144. & & B5 & B9 III & -1.0 & 0.46 & 0.60 & 1.90 & 440 & 3 \\
145. & & & K0 II & -2.6 & 0.80 & 0.94 & 3.36 & 770 & 2 \\
146. & & & F5 V & 3.6 & 0.10 & 0.13 & 0.41 & 220 & 2 \\
147. & 8607 & F0 & F1 III & 1.6 & 0.02 & 0.02 & 0.08 & 260 & 3 \\
148. & & & F4 V & 3.6 & 0.02 & 0.02 & 0.08 & 240 & 3 \\
149. & & A & A0 III & -0.9 & 0.40 & 0.53 & 1.66 & 670 & 2 \\
150. & 8633 & B8 & B9 II & -0.8 & 0.43 & 0.56 & 1.78 & 460 & 3 \\
$151 . *$ & & K5 & K0 III & 0.9 & 0.51 & 0.61 & 2.13 & 190 & 1 \\
152. & 8756 & A3 & B5 IV & -1.2 & 0.10 & 0.13 & 0.41 & 870 & 3 \\
\hline
\end{tabular}

Notes to Table 3:

1. Photometric classification uncertain; interstellar extinction seems to be too high for this distance; unresolved binary?

31. Buscombe (1984) gives M2 III:;

35. Jashek (1978) gives A0 III;

88. Buscombe (1984) gives B5 Vne;

91. Photometric classification uncertain due to low accuracy of the short wavelength indices;

93. Photometric classification uncertain due to low accuracy of the short wavelength indices;

96. ADS $927 \mathrm{C}$ at $37.7^{\prime \prime}$ from the components $\mathrm{A}+\mathrm{B}$;

97. Close binary ADS $927 \mathrm{AB}$; the faint component of $12.5 \mathrm{mag}$ is at $2.8^{\prime \prime}$; both stars were measured together;

104. Binary; the faint component of $12.9 \mathrm{mag}$ is at $15^{\prime \prime}$; only the bright component was measured;

111. Binary; only the bright component was measured;

121. Buscombe (1984) gives A0 Ib;

130. Binary; the faint component of $14.3 \mathrm{mag}$ is at $20^{\prime \prime}$; only the bright component was measured;

143. Binary; the faint component of $14.2 \mathrm{mag}$ is at $17^{\prime \prime}$; only the bright component was measured;

151. Photometric classification uncertain, interstellar extinction seems to be too high for this distance; unresolved binary? 
Fig. 3 shows the run of interstellar extinction in a wide belt with the galactic coordinates $l=120^{\circ}-130^{\circ}$ and $b=+2^{\circ}-+10^{\circ}$. The data are taken from the catalogue of Guarinos (1992), now accessible on Internet. One can see that the main rise of interstellar extinction takes place between 200 and $1000 \mathrm{pc}$. The mean value of interstellar extinction at larger distances is about $2.5 \mathrm{mag}$ and does not grow up with distance. A comparison of Figs. 2 and 3 shows that the investigated area is covered by more opaque clouds than the surrounding region: in our area the maximum value of extinction is $3.4 \mathrm{mag}$.

Some stars in the foreground, closer than $200 \mathrm{pc}$, exhibit too large extinction for their distance. Probably, these stars are either unresolved binaries of close spectral types or peculiar objects. They are marked by asterisks in Table 3 and are not plotted in Fig. 3 .

ACKNOWLEDGMENTS. The author is grateful to V. Straižys for helpful discussions and to V. Laugalys for his assistance in observations. The author thanks the International Science Foundation for a partial support by the grant LE9000.

\section{REFERENCES}

Buscombe W. 1984, MK Spectral Classifications, Northwestern University, Evanston

Guarinos J. 1992, in Astronomy from Large Databases II, eds. A. Heck \& F. Murtagh (ESO Proceedings, Vol. 43), p. 301

Jaschek M. 1978, Catalogue of Selected Spectral Types in the MK System, Inform. Bull. CDS, Strasbourg, No. 15, 121

Haupt H. F., Schroll A. 1974, A\&AS, 15, 311

Khavtassi J.S. 1960, Atlas of Galactic Dark Nebulae, Abastumani

Observatory, Tbilisi

Kurilienè G., Sũdžius J. 1974, Bull. Vilnius Obs., No. 40, 10

Lynds B. T. 1962, ApJS, 7, 1

Neckel H. 1974, A\&AS, 18, 169

Oja T. 1991, A\&AS, 89, 415

Olsen E. H. 1983, A\&AS, 54, 55

Perry C. L., Johnston L. 1982, A\&AS, 50, 451

Pesch P. 1963, ApJ, 137, 547

Straižys V. 1992, Multicolor Stellar Photometry, Pachart Publishing House, Tucson, Arizona

Straižys V., Kurilienė G., Jodinskienė E. 1982, Bull. Vilnius Obs., No. 39,18 
Straižys V., Kazlauskas A. 1993, Baltic Astronomy, 2, 1

Sūdžius J. 1974, Bull. Vilnius Obs., No. 39, 18

Sviderskienė Z. 1988, Bull. Vilnius Obs., No. 80, 3

Vansevičius V., Bridžius A. 1994, Baltic Astronomy, 3, 193

Zdanavičius K. 1975, Bull. Vilnius Obs., No. 41, 3

Zdanavičius K., Claria J.J., Piatti A.E., Straižys V. 1994, Baltic Astronomy, 3, 217

Zdanavičius K., Černienè E. 1985, Bull. Vilnius Obs., No. 69, 3 
